\title{
Research progress of intestinal microecology in the occurrence and development of precancerous lesions of liver
}

\author{
Meng-yang QU, Yan-fang PAN *, Min Xie \\ Department of Basic Medicine, Shaanxi University of Chinese Medicine, Xianyang, Shaanxi 712046, PR China
}

\begin{abstract}
Intestinal microecology refers to the interaction between the host and the microorganisms in the human intestinal tract, which is composed mainly of intestinal flora. Intestinal microflora affects the physiological and pathological changes of the host through metabolic activity and host interaction. Precancerous lesion of liver is a potential benign liver disease, which may lead to malignant transformation of liver. It is the intermediate stage from benign lesion to malignant transformation. Recent studies have shown that intestinal microecology is closely related to the occurrence of precancerous lesions of the liver. This study expounds the interaction of the bridge between intestine and liver, the gutliver axis, the intestinal microecology and the precancerous lesions of liver, hoping to provide a new idea for clinical prevention and treatment of precancerous lesions of liver.
\end{abstract}

\section{Introduction}

Intestinal microecology is a complex and diverse ecosystem, which has a specific symbiotic relationship with the host. At present, intestinal microecology plays an important role in the physiological and pathological process of the host, which is closely related to the pathogenesis of liver diseases ${ }^{[1]}$. The gut microecology includes the gut microbiome, which normally maintains a dynamic equilibrium with the host, but is disrupted by changes in the host and environment, leading to disease ${ }^{[2]}$.

Hepatocellular carcinoma (HCC) is the most common primary liver cancer and the sixth most common malignant tumor in the world, with high malignancy and the third highest mortality rate in the world $^{[3]}$. Hepatiti-liver fibrosis-liver cirrhosis-liver precancerous lesions-liver cancer is the five stages of liver disease development. In the liver cirrhosis, the liver cancer malignant transformation process, will experience a quite long time, namely the liver cancer precancerous lesion stage ${ }^{[4]}$. Because the early symptoms of liver cancer are not obvious and easy to be ignored, and the cure rate of advanced liver cancer is low, the cure difficulty is big and the treatment plan is complex, so it is more meaningful to prevent the occurrence and development of precancerous lesions than to treat liver cancer. Precancerous lesion of liver is a potential benign liver lesion with the possibility of malignant transformation of liver. It is the intermediate stage from benign lesion to malignant transformation. Precancerous lesions of liver mainly include liver cell dysplasia, hepatic adenomatous hyperplasia, liver fibrosis, liver

\footnotetext{
Corresponding author: panyanfang2000@163.com
}

cirrhosis and other diseases. It is influenced by hepatitis, alcoholic liver disease, nonalcoholic fatty liver disease, cirrhosis and many other factors. In recent years, with the further study of the intestinal liver axis, it has been found that the intestinal microecological imbalance and the progression of chronic liver disease can affect each other ${ }^{[5]}$, this article discusses the relationship between Intestinal microecology and the occurrence and development of precancerous lesions of liver, and provides more directions for the prevention and treatment of precancerous lesions of liver.

\section{Gut microecology}

The intestinal microecology is a kind of ecosystem which is composed of the interaction between the microorganism and the host. Its main functions include participating in metabolic activities, nutritional effects, immunity and protection of receptors from foreign microorganisms ${ }^{[6]}$. Dysbacteriosis can lead to inflammation and fibrosis of the liver ${ }^{[6,7]}$. Probing into the composition of intestinal microflora is helpful to understand the relationship between intestinal microecology and host diseases.

\section{Composition of intestinal flora}

The gut microbiome consists of bacteria, archaea, and eukaryotes that congregate in the gastrointestinal tract and have evolved with their hosts over thousands of years to form complex and mutually beneficial interactions ${ }^{[8,9]}$. According to statistics, there are more than 1014 kinds of microorganisms in the digestive tract, among which there are 10 times more bacterial cells and 
100 times more microbiome than human genome ${ }^{[10]}$. Although the gut microbiome was previously thought to consist of between 500 and 1,000 species of microbes ${ }^{[11]}$, a recent large-scale study estimated that the gut microbiome consists of more than 3,500 species of bacteria $^{[12]}$. Intestinal flora can be divided into three categories: beneficial bacteria, pathogenic bacteria and conditional pathogenic bacteria. Beneficial bacteria such as Bifidobacterium, lactobacillus, etc; Pathogenic bacteria such as Staphylococcus, proteus, clostridium, pseudomonas Aeruginosa and opportunistic pathogens such as Enterococcus, Escherichia coli, bacteroides $^{[13]}$.The intestinal microflora can be divided into five groups: bacteroidetes, firmicutes, actinomycetes, proteobacteria and verruculose microphytes. The number of bacteroidetes and firmicutes accounts for more than $90 \%$ of healthy people's intestinal tract, the quantity of Actinomycota, proteobacteria and Verrucosa microbacteria was less ${ }^{[14]}$.

\section{Metabolites of Intestinal Flora}

The metabolic products of the intestinal flora are shortchain fatty acid and secondary bile, in which short-chain fatty acid is the major. Short-chain fatty acid is produced by intestinal bacteria that ferment polysaccharides that the body can not digest. Short-chain fatty acid regulates various aspects of the host by inhibiting histone deacetylase and activating specific $\mathrm{G}$ protein coupled receptors $^{[15]}$, such as intestinal motility, inflammation, glucose homeostasis, and energy. Short-chain fatty acid consists mainly of acetate, propionate and Butyrate, which are important energy sources for intestinal epithelial cells to maintain the intestinal barrier, and propionate, which converts and inhibits cholesterol synthesis in the liver. Intestinal dysbacteriosis is caused by overgrowth of bacteria and abnormal accumulation of metabolic products, which leads to the destruction of intestinal barrier and liver damage caused by large numbers of bacteria entering the liver. Studies have shown that exogenous acetate and propionate can prevent hepatic steatosis, inhibit hepatic fat synthesis, reduce liver damage caused by hepatic steatosis and prevent precancerous liver lesions ${ }^{[16]}$. Short-chain fatty acid can also increase the risk of hepatocellular carcinoma by inducing cholestasis ${ }^{[17]}$.

\section{Intestinal hepatic axis}

The liver is an organ with a dual blood supply. It supplies oxygenated blood to the liver from the celiac artery and receives blood from the intestine to the hepatic portal vein. About $75 \%$ of the liver's blood comes from the hepatic vein. Therefore, there is a close relationship between the intestine and the liver, forming the intestine-liver axis. The enteric-hepatic axis refers to the mutual regulation of the intestinal mucosa and the liver through the portal circulation. The relationship between intestinal flora and liver is regulated and stabilized by the interaction of metabolism, immunity and neuroendocrine ${ }^{[18]}$. Intestinal barrier is a barrier formed by connecting adjacent cells through tight junction protein, which is a natural barrier against bacteria and their metabolites ${ }^{[19]}$. In the absence of the host's gut microbiome, the antigens (derived from pathogenic microorganisms) pass through these tight junctions, which are recognized by the dendritic cell and activate the adaptive immune system, resulting in increased intestinal permeability. Small doses of pathogen-related molecules such as Lipopolysaccharide (Lps), peptidoglycan and flagellin enter the liver via Toll-like receptor $4(\mathrm{Tl} 4)$ and cause liver fibrosis ${ }^{[20]}$.

\section{Intestinal microecology and precancerous lesions of liver}

Changes of intestinal microflora in patients with precancerous lesions of liver. When the liver becomes diseased, the dynamic equilibrium between the host and the gut ecology is broken down, leading to a disruption of the gut flora. Liver fibrosis and cirrhosis may lead to bacterial translocation through different mechanisms, including overgrowth of intestinal bacteria, derangement of intestinal lumen factors, increase of intestinal permeability and decrease of immune function ${ }^{[21]}$. According to research, liver fibrosis and cirrhosis are closely related to intestinal microecological imbalance ${ }^{[2]}$. In cirrhosis, a decrease in bile acid secretion ${ }^{[23]}$ and portal hypertension ${ }^{[24]}$ overgrowth of the intestinal flora. The study showed that the intestinal flora of patients with cirrhosis showed higher levels of pathogenic bacteria such as enterobacteriaceae and streptococci, as well as lower levels of beneficial bacteria such as bifidobacterium and lactobacillus, compared with healthy controls ${ }^{[25,26]}$. Quantitative metagenomic analysis of intestinal microflora in patients with liver.

Intestinal microecological imbalance promotes the occurrence and development of precancerous lesions of liver

Intestinal microecological imbalance refers to increased permeability of intestinal mucosa, a large number of bacterial translocation. Bacterial translocation refers to the migration of live bacteria or bacterial endotoxin (lipopolysaccharide, peptidoglycan, lipopeptide) from the intestinal cavity to the mesenteric lymph nodes and other extraintestinal sites ${ }^{[27]}$. When bacteria overgrow, intestinal permeability increases and a lot of bacterial translocation, especially of lipopolysaccharide (gram-negative), reaches the liver through the hepatic portal vein, it binds to toll-like receptor 4 in various liver cells, such as kupffer cells (KCs) and hepatic stellate cells (HSCs), causing chronic hepatitis, cirrhosis and liver cancer ${ }^{[28]}$. Therefore, the activation of LPS-TLR4 pathway is very important in the development of liver precancerous lesions. When activated by LPS-TLR4, KCs in liver cells secrete TNF$\alpha$, IL-6 and other inflammatory mediators, which cause oxidative stress and inflammatory reaction in liver, damage cell DNA and induce gene mutation ${ }^{[29]}$. At the same time, when HSCs are activated by the LPS-TLR4 
pathway, they can synthesize a large number of extracellular matrix proteins, which can promote liver fibrosis, and further promote the development of liver cirrhosis. After activation, HSCs also secretes angiogenesis factors, IPS induced the migration of mesenchymal endothelial cells and the formation of blood vessels, which is a key step in the development of hepatocellular carcinoma ${ }^{[30]}$. Therefore, the composition and function changes of intestinal microecology may be closely related to the development of liver fibrosis and cirrhosis, thus promoting the development of precancerous liver lesions.

\section{Conclusion}

Intestinal microecological imbalance is closely related to the occurrence and development of precancerous lesions of liver. Precancerous lesions of the liver in patients with intestinal pathogenic bacteria significantly increased and beneficial bacteria significantly reduced, at the same time, the imbalance of intestinal microecology leads to the overgrowth of intestinal bacteria, the increase of intestinal permeability and bacterial translocation, which induce a lot of lipopolysaccharide to enter the liver through the portal vein. Therefore, the composition of intestinal flora, metabolic products, prevent bacterial translocation, block the signal pathway may delay or prevent the development of precancerous liver lesions. At present, there are few studies on the relationship between intestinal microecology and precancerous lesions of liver. Therefore, it is necessary to explore the relationship between intestinal microecology and liver precancerous lesion to provide a new therapeutic idea for liver precancerous lesion.

\section{Acknowledgements}

This research was supported by the National Science Foundation of PR China (81703842) and Key Research and Development Projects of Shaanxi Provincial (2020SF-343) and (2018SF-036)

\section{Reference}

1. Ann MO, Fergus S. The gut flora as a forgotten organ. EMBO reports, 2006, 7(7): 688-693.

2. Z Wang Z, Klipfell E, Bennett B J , et al. Gut flora metabolism of phosphatidylcholine promotes cardiovascular disease[J]. Nature, 2011, 472(7341):57-63.

3. Davila J A , El-Serag H . 458 The Rising Incidence of Hepatocellular Carcinoma in the United States: an Update[J]. Gastroenterology, 2012, 142(5):S914-S-914.

4. Luca DT, Angelo S, Mauro B, et al. Advanced precancerous lesionsin the liver $[\mathrm{J}]$. Best
Practice $R$ esearch Clinical Gzstroenterology , 2013, 27(2): $269-284$.

5. Milosericl I, Vujovic A, Barac A, et al. Gutliver axis, gut microbiota, and its modulation in the management of liver diseases: a review of the literature . International Journal of Molecular Sciences, 20.2 (2019): 395.

6. Jorge HM, Eran E, Chengcheng J, et al . Inflammasome-mediated dysbiosis regulates progression of NAFLD and obesity. Nature, 2012, 482: 179-85.

7. Kouichi M, Hirohide O. Role of gut microbiota and Toll-like receptors in nonalcoholic fatty liver disease. World J Gastroenterol, 2014, 20: 738191.

8. Fredrik B, Ruth EL, Justin LS, et al. Hostbacterial mutualism in the human intestine. Science, 2005, 307(5717) : 19151920 .

9. Andrew SN. Microbes in gastrointestinal health and disease. Gastroenterology, 2009, 136(1): 65-80.

10. Gill SR , Pop M , DeBoy RT, et al. Metagenomic analysis of the human distal gut microbiome. Science, 2006, 312: 1355-1359.

11. Ramakrishna B, Krishnan S. The normal bacterial flora of the human intestine and its regulation. Journal of Clinical Gastroenterology, 2007, 41(Supplement 1): S2-S6.

12. Daniel NF, Allison LSA, Robert AF, et al . Molecular-phylogenetic characterization of microbial community imbalances in human inflammatory bowel diseases. Proc Natl Acad Sci USA, 2007, 104: 13780-13785.

13. Junjie Q, Ruiqiang L, Jeroen R, et al. A human gut microbial gene catalogue established by metagenomics by metagenomics sequencing[J]. Nature, 2010, 464 (7285): 5965.

14. Sebastian D, Juan J, Sanchez S, et al. From the intestinal flora to the microbiome[J]. Rev Esp Enferm Dig, 2018, 110(1): 51-

15. Sharon G, Garg N, Debelius J , et al. Specialized metabolites from the microbiome in health and disease[J]. Cell Metab, 2014, 20 (5) : 719-730.

16. Weitkunat K, Stuhlmann C, Postel A, et al. Short-chain fatty acids and inulin, but not guar gum, prevent diet-induced obesity and insulin resistance through differential mechanisms in mice[J]. Sci Rep, 2017, 7(1): 6109. 
17. Suchira G, Elena K, Mathias H, et al. When Soluble Fibers Meet Hepatocellular Carcinoma: The Dark Side of Fermentation[J] . Cell Metab , 2018, 28(5): 673-675.

18. Zhi YK , Sunil KL . The Human Gut Microbiome-A Potential Controller of Wellness and Disease[J] . other, 2018, 9: 1835.

19. Pietro V, Giulia P, Alessio F. Microbiota and gut-liver axis: A mini-review on their influences on obesity and obesity-related liver disease[J]. Journal of Pediatric Gastroenterology \& Nutrition, 2013, 56(5): 461-468.

20. Yiu JH, Dorweiler B, Woo CW. Interaction between gut microbiota and toll-like receptor: From immunity to metabolism[J] . Journal of Molecular Medicine, 2017, 95(1): 13-20.

21. Marilia RP, Benedetto MC, Michele DR, et al. Microbial Translocation in Chronic Liver Diseases[J]. International Journal of Microbiology, 2012, 2012(2):694629.

22. Bajaj JS , Heuman DM, Hylemon PB , etal. Altered profile of human gut microbiome is associated with cirrhosis and its complications $[\mathrm{J}]$. Journal of Hepatology, 2014, 60(5): 940-947.

23. Ekihiro S, Bernd S. Role of innate immunity and the microbiota in liver fibrosis: crosstalk between the liver and gut[J] . Journal of Physiology, 2012, 590(3): 447-458.

24. Dupont A W, Dupont H L . The intestinal microbiota and chronic disorders of the gut[J] . Nature Reviews Gastroenterology \& Hepatology, 2011, 8(9): 523-31.

25. Chen Y, Yang F, Lu H, et al. Characterization of fecal microbial communities in patients with liver cirrhosis. Hepatology, 2011, 54: 562-572.

26. Haifeng L, Zhongwen W, Wei X, et al. Intestinal Microbiota Was Assessed in Cirrhotic Patients with Hepatitis B Virus Infection[J]. Microbial Ecology, 2011, 61(3): 693-703.

27. Daniel B, Reiner W. Gut microbiome and intestinal barrier failure--the " Achilles heel " in hepatology? [J]. Journal of Hepatology, 2012, 56(6): 1221-1223

28. Brandi G, De L , Candela M , et al. Microbiota, NASH, HCCand the potential role of probiotics[J] . Carcinogenesis , 2017, 38(3): 231-240.

29. Yu LX, Schwabe RF. The gut microbiome and liver cancer : mecha-nisms and clinical
translation[J]. Nat Rev Gastroenterol Hepatol, 2017, 14(9): 527-539.

30. Lijun W, Yujui YW. The role of gut microbiota in liver disease devel-opment and treatment[J]. Liver Res, 2019, 3(1): 3-18. 